\title{
On the number of representations of a positive integer by a binary quadratic form
}

by

Pierre Kaplan (Nancy) and Kenneth S. Williams (Ottawa)

0. Notation. Throughout this paper $n$ denotes a positive integer and $d$ denotes a discriminant, that is, $d$ is a nonsquare integer such that $d \equiv 0$ or $1(\bmod 4)$. We set

$$
w(d)= \begin{cases}6 & \text { if } d=-3 \\ 4 & \text { if } d=-4 \\ 2 & \text { if } d<-4 \\ 1 & \text { if } d>0\end{cases}
$$

If $d>0$ we let

$$
\varepsilon(d)=\frac{1}{2}\left(x_{0}+y_{0} \sqrt{d}\right),
$$

where $\left(x_{0}, y_{0}\right)$ is the solution of $x^{2}-d y^{2}=4$ in positive integers with $y$ least. If $m$ is a positive integer such that $m^{2} \mid d$ and $d / m^{2}$ is a discriminant, we set

$$
\lambda(d, m)= \begin{cases}1 & \text { if } d<0, \\ \frac{\log \varepsilon(d)}{\log \varepsilon\left(d / m^{2}\right)} & \text { if } d>0 .\end{cases}
$$

The conductor $f=f(d)$ of the discriminant $d$ is the largest positive integer $f$ such that $d / f^{2}$ is a discriminant. The discriminant $\Delta=\Delta(d)=d / f(d)^{2}$ is called the fundamental discriminant associated with $d$. If $f(d)=1$, then $d$ is called fundamental. We denote by $M=M(n, d)$ the largest positive integer $M$ such that $M^{2} \mid n$ and $M \mid f$. Equivalently $M$ is the largest positive integer such that $M^{2}\left|n, M^{2}\right| d$ and $d / M^{2}$ is a discriminant.

2000 Mathematics Subject Classification: Primary 11E16, 11E25.

Key words and phrases: representations of an integer by a binary quadratic form.

Research of the second author was supported by Natural Sciences and Engineering Research Council of Canada grant A-7233. 
Let $(a, b, c)=a x^{2}+b x y+c y^{2}$ be a primitive, integral, binary quadratic form of discriminant $d$, which is irreducible in $\mathbb{Z}[x, y]$, so that $a, b, c$ are integers such that $\operatorname{gcd}(a, b, c)=1$ and $d=b^{2}-4 a c \equiv 0$ or $1(\bmod 4)$ is not a square in $\mathbb{Z}$. If $d<0$ we only consider positive-definite forms, that is, forms $(a, b, c)$ with $a>0$. The positive integer $n$ is said to be represented by the form $(a, b, c)$ if there exists $(x, y) \in \mathbb{Z} \times \mathbb{Z}$ such that $n=a x^{2}+b x y+c y^{2}$. In the case $d<0$ every representation $(x, y)$ of $n$ by $(a, b, c)$ is said to be primary. In the case $d>0$ only those representations $(x, y)$ of $n$ by $(a, b, c)$ are called primary that satisfy the inequalities

$$
2 a x+(b-\sqrt{d}) y>0, \quad 1 \leq\left|\frac{2 a x+(b+\sqrt{d}) y}{2 a x+(b-\sqrt{d}) y}\right|<\varepsilon(d)^{2} .
$$

The number of primary representations of $n$ by the form $(a, b, c)$ is denoted by $R_{(a, b, c)}(n, d)$. It is known that $R_{(a, b, c)}(n, d)$ is finite. The class of the form $(a, b, c)$ is the set $[a, b, c]$ given by

$$
\begin{array}{r}
{[a, b, c]=\left\{a(p x+q y)^{2}+b(p x+q y)(r x+s y)+c(r x+s y)^{2} \mid\right.} \\
p, q, r, s \in \mathbb{Z}, p s-q r=1\} .
\end{array}
$$

The set of distinct classes of primitive, integral, binary quadratic forms of discriminant $d$ is denoted by $H(d)$. Let $K \in H(d)$. If $[a, b, c]=\left[a^{\prime}, b^{\prime}, c^{\prime}\right]=K$ then $R_{(a, b, c)}(n, d)=R_{\left(a^{\prime}, b^{\prime}, c^{\prime}\right)}(n, d)$ so we can define

$$
R_{K}(n, d)=R_{(a, b, c)}(n, d) \quad \text { for any }(a, b, c) \in K .
$$

With respect to Gaussian composition (see for example [2, Chapter 4]), $H(d)$ is a finite abelian group called the form class group. The order of $H(d)$ is called the form class number and is denoted by $h(d)$. The genus group of $H(d)$ is the quotient group $G(d)=H(d) / H^{2}(d)$. It is known that $|G(d)|=2^{t(d)}$, where $t(d)$ is the nonnegative integer given by

$$
t(d)= \begin{cases}\omega(d)-2 & \text { if } d \equiv 4(\bmod 16) \\ \omega(d) & \text { if } d \equiv 0(\bmod 32) \\ \omega(d)-1 & \text { otherwise }\end{cases}
$$

where $\omega(d)$ is the number of distinct prime factors of $d$. The number of form classes in each genus $G \in G(d)$ is $h(d) / 2^{t(d)}$. For $G \in G(d)$ we set

$$
R_{G}(n, d)=\sum_{K \in G} R_{K}(n, d) .
$$

An odd prime discriminant is a discriminant of the form $p^{*}=(-1)^{(p-1) / 2} p$, where $p$ is an odd prime. The discriminants $-4,8,-8$ are called even prime discriminants. Set $t=t(d)$, where $t(d)$ is defined in (0.4). The prime discriminants corresponding to the discriminant $d$ are the discriminants 
$p_{1}^{*}, \ldots, p_{t+1}^{*}$, together with $p_{t+2}^{*}$ if $d \equiv 0(\bmod 32)$, where $p_{1}, \ldots, p_{t+1}$ are given as follows:

(i) $d \equiv 1(\bmod 4)$ or $d \equiv 4(\bmod 16)$

$p_{1}<\ldots<p_{t+1}$ are the odd prime divisors of $d$.

(ii) $d \equiv 12(\bmod 16)$ or $d \equiv 16(\bmod 32)$

$p_{1}<\ldots<p_{t}$ are the odd prime divisors of $d$ and $p_{t+1}^{*}=-4$.

(iii) $d \equiv 8(\bmod 32)$

$p_{1}<\ldots<p_{t}$ are the odd prime divisors of $d$ and $p_{t+1}^{*}=8$.

(iv) $d \equiv 24(\bmod 32)$

$p_{1}<\ldots<p_{t}$ are the odd prime divisors of $d$ and $p_{t+1}^{*}=-8$.

(v) $d \equiv 0(\bmod 32)$

$p_{1}<\ldots<p_{t-1}$ are the odd prime divisors of $d, p_{t}^{*}=-4$,

$p_{t+1}^{*}=8$, and $p_{t+2}^{*}=-8$.

The set of prime discriminants corresponding to $d$ is denoted by $P(d)$. We note that these are coprime in pairs if $d \not \equiv 0(\bmod 32)$. The set of all products of pairwise coprime elements of $P(d)$ is denoted by $F(d)$. The empty product 1 is included in $F(d)$. Thus, for example, with $d=384=2^{7} \cdot 3$, we have

$$
t(d)=2, \quad p_{1}=3, \quad p_{1}^{*}=-3, \quad p_{2}^{*}=-4, \quad p_{3}^{*}=8, \quad p_{4}^{*}=-8,
$$

and

$$
P(d)=\{-3,-4,8,-8\}, \quad F(d)=\{1,-3,-4,8,-8,12,-24,24\} .
$$

Properties of the sets $P(d)$ and $F(d)$ are given in [4, Lemma 2.1, p. 277] and [9, Lemma 1, p. 29].

Let $p^{*} \in P(d)$ and $K \in H(d)$. For any positive integer $k$ which is coprime with $p^{*}$ and represented by $K$, it is known that $\left(\frac{p^{*}}{k}\right)$ has the same value so we can set

$$
\gamma_{p^{*}}(K)=\left(\frac{p^{*}}{k}\right)= \pm 1
$$

A main result of Gauss's theory of genera is that the genera are the sets of classes in $H(d)$ giving the same value to $\gamma_{p^{*}}$ for each $p^{*} \in P(d)$. Thus, for each genus $G \in G(d)$ and each $p^{*} \in P(d)$, we can set $\gamma_{p^{*}}(G)=\gamma_{p^{*}}(K)$, where $K$ is any class in $G$. The definition of $\gamma_{p^{*}}(G)\left(p^{*} \in P(d)\right)$ is extended to $\gamma_{d_{1}}(G)\left(d_{1} \in F(d)\right)$ by

$$
\gamma_{d_{1}}(G)=\prod_{p^{*} \in P\left(d_{1}\right)} \gamma_{p^{*}}(G)=\left(\frac{d_{1}}{a}\right)= \pm 1,
$$

where the class $[a, b, c] \in G$ is chosen so that $a$ is prime to $d$.

A prime $p$ is said to be a null prime with respect to $n$ and $d$ if

$$
v_{p}(n) \equiv 1(\bmod 2), \quad v_{p}(n)<2 v_{p}(f),
$$


where $p^{v_{p(k)}}$ is the largest power of $p$ dividing the positive integer $k$. The set of all such null primes is denoted by $\operatorname{Null}(n, d)$. The following elementary result is proved in [4, Proposition 4.1] for $d<0$ and for both positive and negative $d$ in [9, Lemma 5].

Proposition 1. If $\operatorname{Null}(n, d) \neq \emptyset$ then $R_{K}(n, d)=0$ for each $K \in$ $H(d)$.

The following result is proved in $[4$, Theorem 8.1, p. 289] in the case $d<0$ and in [9, Theorem 1, p. 38] in the case $d>0$.

Proposition 2. Let $G \in G(d)$. If $\operatorname{Null}(n, d)=\emptyset$ then

$$
\begin{aligned}
R_{G}(n, d)= & \lambda(d, M) \frac{h(d)}{h\left(d / M^{2}\right)} \frac{w\left(d / M^{2}\right)}{2^{t(d)+1}} \\
& \times \sum_{d_{1} \in F\left(d / M^{2}\right)} \gamma_{d_{1}}(G) \sum_{\mu \nu=n / M^{2}}\left(\frac{d_{1}}{\mu}\right)\left(\frac{d / M^{2} d_{1}}{\nu}\right) .
\end{aligned}
$$

If $\operatorname{Null}(n, d) \neq \emptyset$ then $R_{G}(n, d)=0$.

The proof of Proposition 2 follows from Gauss's theory of genera and so is an elementary theorem.

1. Introduction. From Proposition 2 it is possible to give a formula for $R_{(a, b, c)}(n, d)$ when $[a, b, c]$ belongs to a genus consisting of exactly one class or consisting of exactly two classes $K$ and $K^{-1}$ with $K \neq K^{-1}$. A formula for $R_{(a, b, c)}(n, d)$ when $[a, b, c]$ belongs to a genus consisting of exactly one class has been given by Hall [3]. No formula is known for $R_{(a, b, c)}(n, d)$ for an arbitrary form $(a, b, c)$. However, a number of authors (for example van der Blij [1], Lomadze [6]-[8], Vepkhvadze [10]-[14]) have obtained formulae for $R_{(a, b, c)}(n, d)$ for certain special forms $a x^{2}+b x y+c y^{2}$, which belong to genera having at least three classes or consisting of exactly two classes $K_{1}$ and $K_{2}$ with $K_{1} \neq K_{2}, K_{1}=K_{1}^{-1}, K_{2}=K_{2}^{-1}$. In most cases their formulae for $R_{(a, b, c)}(n, d)$ have $d$ nonfundamental and depend upon the coefficients in the expansion of certain products of theta functions. For example Lomadze [7, Theorem 7a] proved

$$
R_{(1,0,32)}(n,-128)= \begin{cases}\sum_{d \mid n}\left(\frac{-2}{d}\right)+\frac{1}{2} v(n) & \text { if } \alpha=0, u \equiv 1(\bmod 8), \\ 2 \sum_{d \mid n}\left(\frac{-2}{d}\right) & \text { if } \alpha=2, u \equiv 1(\bmod 8), \\ 0 & \text { or } \alpha>3, u \equiv 1 \operatorname{or} 3(\bmod 8),\end{cases}
$$

where $n=2^{\alpha} u, u$ odd, and $v(n)$ denotes the coefficient of $Q^{n}$ in the expansion of the function $\theta_{80}(\tau ; 0,8) \theta_{01}(\tau ; 0,16)$ in powers of $Q=\exp (2 \pi i \tau)$, 
where

$$
\theta_{g h}(\tau ; c, N)=\sum_{\substack{m=-\infty \\ m \equiv c(\bmod N)}}^{\infty}(-1)^{h(m-c) / N} Q^{(m+g / 2)^{2} / 2 N} .
$$

We note that the second and third lines of the formulae for $R_{(1,0,32)}(n,-128)$ do not depend upon the quantity $v(n)$. They depend at most on the values of the Kronecker symbol $\left(\frac{-2}{d}\right)$ for $d \mid n$ and thus are elementary formulae even though they were derived by advanced analytical techniques. In this paper we prove a general theorem by entirely elementary means from which these and other elementary formulae follow as special cases.

\section{Statement and proof of main result}

THEOREM 1. Let $d$ be a discriminant for which there exists a positive integer $m$ such that $d / m^{2}$ is a discriminant and either

$$
H\left(d / m^{2}\right)=\mathbb{Z}_{2} \times \ldots \times \mathbb{Z}_{2}
$$

or

$$
H\left(d / m^{2}\right)=\mathbb{Z}_{4} \times \mathbb{Z}_{2} \times \ldots \times \mathbb{Z}_{2} \quad \text { (Case II) } .
$$

Let $K=[a, b, c] \in H(d)$ with $a, b, c$ chosen so that

$$
(a, d)=1, \quad m\left|b, \quad m^{2}\right| c .
$$

Let $n$ be a positive integer. If $\operatorname{Null}(n, d) \neq \emptyset$ then $R_{K}(n, d)=0$. If $\operatorname{Null}(n, d)$ $=\emptyset$ and $m^{2} \mid n$ then in Case I

$$
\begin{aligned}
R_{K}(n, d)= & \lambda(d, M) \frac{w\left(d / M^{2}\right)}{2^{t\left(d / m^{2}\right)+1}} \frac{h\left(d / m^{2}\right)}{h\left(d / M^{2}\right)} \\
& \times \sum_{d_{1} \in F\left(d / M^{2}\right)}\left(\frac{d_{1}}{a}\right) \sum_{\mu \nu=n / M^{2}}\left(\frac{d_{1}}{\mu}\right)\left(\frac{d / M^{2} d_{1}}{\nu}\right)
\end{aligned}
$$

and in Case II

$$
\begin{aligned}
R_{K}(n, d)= & \lambda(d, M) \frac{w\left(d / M^{2}\right)}{2^{t\left(d / m^{2}\right)+2}} \frac{h\left(d / m^{2}\right)}{h\left(d / M^{2}\right)} \\
& \times \sum_{d_{1} \in F\left(d / M^{2}\right)}\left(\frac{d_{1}}{a}\right) \sum_{\mu \nu=n / M^{2}}\left(\frac{d_{1}}{\mu}\right)\left(\frac{d / M^{2} d_{1}}{\nu}\right)
\end{aligned}
$$

provided $\left[a, b / m, c / m^{2}\right] \neq\left[a, b / m, c / m^{2}\right]^{-1}$.

Proof. If $\operatorname{Null}(n, d) \neq \emptyset$ then, by Proposition 1 , we have $R_{K}(n, d)=0$. Hence we may suppose that $\operatorname{Null}(n, d)=\emptyset$. Let $m$ be a positive integer such that $d / m^{2}$ is a discriminant and $m^{2} \mid n$. By (0.7) we have

$$
\operatorname{Null}\left(n / m^{2}, d / m^{2}\right)=\emptyset \text {. }
$$


As $m$ is a positive integer such that $m^{2}\left|n, m^{2}\right| d$ and $d / m^{2}$ is a discriminant then $m \mid M=M(n, d)$ and

$$
M\left(n / m^{2}, d / m^{2}\right)=\frac{M(n, d)}{m} .
$$

Hence

$$
\begin{aligned}
& \frac{d / m^{2}}{M\left(n / m^{2}, d / m^{2}\right)^{2}}=\frac{d / m^{2}}{(M(n, d) / m)^{2}}=\frac{d}{M(n, d)^{2}}=\frac{d}{M^{2}}, \\
& \frac{n / m^{2}}{M\left(n / m^{2}, d / m^{2}\right)^{2}}=\frac{n / m^{2}}{(M(n, d) / m)^{2}}=\frac{n}{M(n, d)^{2}}=\frac{n}{M^{2}} .
\end{aligned}
$$

Applying [4, Lemma 6.2, p. 286] in the case $d<0$ and [9, Lemma 14, p. 35] in the case $d>0$ to each prime dividing $m$, we obtain

$$
R_{K}(n, d)=\lambda(d, m) R_{\left[a, b / m, c / m^{2}\right]}\left(n / m^{2}, d / m^{2}\right) .
$$

Let $G \in G\left(d / m^{2}\right)$ be the genus to which the class $\left[a, b / m, c / m^{2}\right]$ belongs. By (2.4)-(2.7) and Proposition 2, we obtain

$$
\begin{aligned}
R_{G}\left(n / m^{2}, d / m^{2}\right)= & \lambda\left(d / m^{2}, M / m\right) \frac{w\left(d / M^{2}\right)}{2^{t\left(d / m^{2}\right)+1}} \frac{h\left(d / m^{2}\right)}{h\left(d / M^{2}\right)} \\
& \times \sum_{d_{1} \in F\left(d / M^{2}\right)} \gamma_{d_{1}}(G) \sum_{\mu \nu=n / M^{2}}\left(\frac{d_{1}}{\mu}\right)\left(\frac{d / M^{2} d_{1}}{\nu}\right) .
\end{aligned}
$$

Now from (0.3) we deduce

$$
\lambda(d, m) \lambda\left(d / m^{2}, M / m\right)=\lambda(d, M)
$$

so that by (0.6) the equation (2.9) becomes

$$
\begin{aligned}
R_{G}\left(n / m^{2}, d / m^{2}\right)= & \frac{\lambda(d, M)}{\lambda(d, m)} \frac{w\left(d / M^{2}\right)}{2^{t\left(d / m^{2}\right)+1}} \frac{h\left(d / m^{2}\right)}{h\left(d / M^{2}\right)} \\
& \times \sum_{d_{1} \in F\left(d / M^{2}\right)}\left(\frac{d_{1}}{a}\right) \sum_{\mu \nu=n / M^{2}}\left(\frac{d_{1}}{\mu}\right)\left(\frac{d / M^{2} d_{1}}{\nu}\right) .
\end{aligned}
$$

We now assume that either Case I or Case II holds.

Case I. As $H\left(d / m^{2}\right)=\mathbb{Z}_{2} \times \ldots \times \mathbb{Z}_{2}$ we have $G=\left\{\left[a, b / m, c / m^{2}\right]\right\}$. The asserted formula follows from (2.8) and (2.11).

Case II. As $H\left(d / m^{2}\right)=\mathbb{Z}_{4} \times \mathbb{Z}_{2} \times \ldots \times \mathbb{Z}_{2}$ and since $\left[a, b / m, c / m^{2}\right] \neq$ $\left[a, b / m, c / m^{2}\right]^{-1}$ we have

$$
G=\left\{\left[a, b / m, c / m^{2}\right],\left[a,-b / m, c / m^{2}\right]\right\} .
$$

Hence

$$
\begin{aligned}
R_{G}\left(n / m^{2}, d / m^{2}\right)= & R_{\left[a, b / m, c / m^{2}\right]}\left(n / m^{2}, d / m^{2}\right) \\
& +R_{\left[a,-b / m, c / m^{2}\right]}\left(n / m^{2}, d / m^{2}\right) .
\end{aligned}
$$


Clearly

$$
R_{\left[a, b / m, c / m^{2}\right]}\left(n / m^{2}, d / m^{2}\right)=R_{\left[a,-b / m, c / m^{2}\right]}\left(n / m^{2}, d / m^{2}\right)
$$

so that

$$
R_{\left[a, b / m, c / m^{2}\right]}\left(n / m^{2}, d / m^{2}\right)=\frac{1}{2} R_{G}\left(n / m^{2}, d / m^{2}\right) .
$$

The asserted formula now follows from (2.8), (2.11) and (2.12).

We note that when $\operatorname{Null}(n, d)=\emptyset$ and $m^{2} \mid n$, the formula for $R_{[a, b, c]}(n, d)$ depends only upon $n, d$ and the genus to which $\left[a, b / m, c / m^{2}\right]$ belongs. In this connection see [15].

3. Some special cases of Theorem 1. The values of $d$ with $-140 \leq$ $d<0$ to which the theorem applies are listed in Table 1 .

Table 1

\begin{tabular}{ccccccc}
\hline$d$ & $H(d)$ & $m$ & $d / m^{2}$ & $H\left(d / m^{2}\right)$ & Corollary & Reference \\
\hline-44 & $\mathbb{Z}_{3}$ & 2 & -11 & $\mathbb{Z}_{1}$ & 1 & {$[7$, Theorem 1a $]$} \\
\hline-63 & $\mathbb{Z}_{4}$ & 3 & -7 & $\mathbb{Z}_{1}$ & 2 & \\
\hline-76 & $\mathbb{Z}_{3}$ & 2 & -19 & $\mathbb{Z}_{1}$ & 3 & {$[7$, Theorem 5a $]$} \\
\hline-80 & $\mathbb{Z}_{4}$ & 2 & -20 & $\mathbb{Z}_{2}$ & 4 & {$[7$, Theorems 3a, 4a $]$} \\
\hline-108 & $\mathbb{Z}_{3}$ & 2 & -27 & $\mathbb{Z}_{1}$ & 5 & \\
-108 & $\mathbb{Z}_{3}$ & 3 & -12 & $\mathbb{Z}_{1}$ & & \\
-108 & $\mathbb{Z}_{3}$ & 6 & -3 & $\mathbb{Z}_{1}$ & & \\
\hline-128 & $\mathbb{Z}_{4}$ & 2 & -32 & $\mathbb{Z}_{2}$ & 6 & {$[7$, Theorem 7a] } \\
-128 & $\mathbb{Z}_{4}$ & 4 & -8 & $\mathbb{Z}_{1}$ & & \\
\hline-135 & $\mathbb{Z}_{6}$ & 3 & -15 & $\mathbb{Z}_{2}$ & 7 & \\
\hline-140 & $\mathbb{Z}_{6}$ & 2 & -35 & $\mathbb{Z}_{2}$ & 8 & \\
\hline
\end{tabular}

Applying the theorem to the discriminants in the table, we obtain the following corollaries. The value of $R_{K}(n, d)$ is only given for those $K \in H(d)$ such that $K=K^{-1}$ and $K$ belongs to a genus containing exactly two classes or $K$ belongs to a genus having three or more classes.

Corollary 1. Let $n=2^{\alpha} 11^{\beta} N$, where $(N, 22)=1$. Then, for $\alpha \geq 1$, we have

$$
\begin{aligned}
R_{[1,0,11]}(n,-44) & =R_{[3, \pm 2,4]}(n,-44) \\
& =\frac{1}{2}\left(1+(-1)^{\alpha}\right)\left(1+\left(\frac{N}{11}\right)\right) \sum_{d \mid N}\left(\frac{d}{11}\right) .
\end{aligned}
$$


Corollary 2. Let $n=3^{\alpha} 7^{\beta} N$, where $(N, 21)=1$. Then, for $\alpha \geq 1$, we have

$$
\begin{aligned}
R_{[1,1,16]}(n,-63) & =R_{[4,1,4]}(n,-63) \\
& =\frac{1}{2}\left(1+(-1)^{\alpha}\right)\left(1+\left(\frac{N}{7}\right)\right) \sum_{d \mid N}\left(\frac{d}{7}\right) .
\end{aligned}
$$

Corollary 3. Let $n=2^{\alpha} 19^{\beta} N$, where $(N, 38)=1$. Then, for $\alpha \geq 1$, we have

$$
\begin{aligned}
R_{[1,0,19]}(n,-76) & =R_{[4, \pm 2,5]}(n,-76) \\
& =\frac{1}{2}\left(1+(-1)^{\alpha}\right)\left(1+\left(\frac{N}{19}\right)\right) \sum_{d \mid N}\left(\frac{d}{19}\right) .
\end{aligned}
$$

Corollary 4. Let $n=2^{\alpha} 5^{\beta} N$, where $(N, 10)=1$. Then, for $\alpha \geq 1$, we have

$$
\begin{aligned}
& R_{[1,0,20]}(n,-80)=R_{[4,0,5]}(n,-80) \\
&= \begin{cases}\frac{1}{2}\left(1+(-1)^{\alpha}\left(\frac{-1}{N}\right)\right)\left(1+\left(\frac{-5}{N}\right)\right) \sum_{d \mid N}\left(\frac{d}{5}\right) & \text { if } \alpha \geq 2, \\
0 & \text { if } \alpha=1 .\end{cases}
\end{aligned}
$$

Corollary 5. Let $n=2^{\alpha} 3^{\beta} N$, where $(N, 6)=1$. Then, for $(\alpha, \beta) \neq$ $(0,0)$, we have

$$
\begin{aligned}
R_{[1,0,27]}(n,-108) & =R_{[4, \pm 2,7]}(n,-108) \\
& =\frac{\theta}{2}\left(1+(-1)^{\alpha}\right)\left(1+\left(\frac{N}{3}\right)\right) \sum_{d \mid N}\left(\frac{d}{3}\right),
\end{aligned}
$$

where

$$
\theta= \begin{cases}0 & \text { if } \alpha=1 \text { or } \beta=1, \\ 1 & \text { if } \alpha=0, \beta \geq 2 \text { or } \alpha \geq 2, \beta=0, \\ 3 & \text { if } \alpha \geq 2 \text { and } \beta \geq 2 .\end{cases}
$$
have

Corollary 6. Let $n=2^{\alpha} N$, where $(N, 2)=1$. Then, for $\alpha \geq 1$, we

$$
\begin{aligned}
R_{[1,0,32]}(n,-128)=R_{[4,4,9]}(n,-128) & \begin{cases}\left(1+\left(\frac{-2}{N}\right)\right) \sum_{d \mid N}\left(\frac{-2}{d}\right) & \text { if } \alpha \geq 4, \\
\frac{1}{2}\left(1+\left(\frac{-1}{N}\right)\right)\left(1+\left(\frac{-2}{N}\right)\right) \sum_{d \mid N}\left(\frac{-2}{d}\right) & \text { if } \alpha=2, \\
0 & \text { if } \alpha=1 \text { or } 3 .\end{cases}
\end{aligned}
$$


Corollary 7. Let $n=3^{\alpha} 5^{\beta} N$, where $(N, 15)=1$. Then, for $\alpha \geq 1$, we have

$$
\begin{aligned}
& R_{[1,1,34]}(n,-135)=R_{[4, \pm 3,9]}(n,-135) \\
& = \begin{cases}\frac{1}{2}\left(1+(-1)^{\alpha+\beta}\left(\frac{N}{5}\right)\right)\left(1+\left(\frac{N}{15}\right)\right) \sum_{d \mid N}\left(\frac{d}{15}\right) & \text { if } \alpha \geq 2, \\
0 & \text { if } \alpha=1,\end{cases} \\
& R_{[2, \pm 1,17]}(n,-135)=R_{[5,5,8]}(n,-135) \\
& = \begin{cases}\frac{1}{2}\left(1-(-1)^{\alpha+\beta}\left(\frac{N}{5}\right)\right)\left(1+\left(\frac{N}{15}\right)\right) \sum_{d \mid N}\left(\frac{d}{15}\right) & \text { if } \alpha \geq 2, \\
0 & \text { if } \alpha=1 .\end{cases}
\end{aligned}
$$

Corollary 8. Let $n=2^{\alpha} 5^{\beta} 7^{\gamma} N$, where $(N, 70)=1$. Then, for $\alpha \geq 1$, we have

$$
\begin{aligned}
& R_{[1,0,35]}(n,-140)=R_{[4, \pm 2,9]}(n,-140) \\
& \quad=\frac{1}{4}\left(1+(-1)^{\alpha}\right)\left(1+(-1)^{\beta+\gamma}\left(\frac{N}{7}\right)\right)\left(1+\left(\frac{N}{35}\right)\right) \sum_{d \mid N}\left(\frac{d}{35}\right), \\
& R_{[3, \pm 2,12]}(n,-140)=R_{[5,0,7]}(n,-140) \\
& \quad=\frac{1}{4}\left(1+(-1)^{\alpha}\right)\left(1-(-1)^{\beta+\gamma}\left(\frac{N}{7}\right)\right)\left(1+\left(\frac{N}{35}\right)\right) \sum_{d \mid N}\left(\frac{d}{35}\right) .
\end{aligned}
$$

Vepkhvadze [16] gave formulae for $R_{[1,0,19]}(n,-76)$ and $R_{[4, \pm 2,5]}(n,-76)$. When $\alpha \geq 1$ his formulae agree with those of Corollary 3. However when $\alpha=0$ his formulae are not correct, as was noted by Zhuravlev [17] in his review of Vepkhvadze's paper. We correct and extend Vepkhvadze's formulae in the next theorem.

VEPKHVADZE'S THEOREM (corrected and extended). Let $m \in\{11,19,27$, $43,67,163\}$. Let $p$ denote the unique prime dividing $m$. Let $m^{*}=\frac{1}{4}(m+1)$. Let $n=p^{\beta} N$, where $(N, 2 p)=1$. Then

$$
\begin{aligned}
R_{[1,0, m]}(n,-4 m) & =-\sum_{x_{1}^{2}+x_{1} x_{2}+m^{*} x_{2}^{2}=n}(-1)^{x_{1}}, \\
R_{\left[4, \pm 2, m^{*}\right]}(n,-4 m) & =\sum_{d \mid N}\left(\frac{-p}{d}\right)+\frac{1}{2} \sum_{x_{1}^{2}+x_{1} x_{2}+m^{*} x_{2}^{2}=n}(-1)^{x_{1}} .
\end{aligned}
$$

Proof. First we note that

$$
R_{\left[4,2, m^{*}\right]}(n,-4 m)=\sum_{4 x_{1}^{2}+2 x_{1} x_{2}+m^{*} x_{2}^{2}=n} 1=\frac{1}{2} \sum_{x_{1}^{2}+x_{1} x_{2}+m^{*} x_{2}^{2}=n}\left(1+(-1)^{x_{1}}\right)
$$




$$
=\frac{1}{2} R_{\left[1,1, m^{*}\right]}(n,-m)+\frac{1}{2} \sum_{x_{1}^{2}+x_{1} x_{2}+m^{*} x_{2}^{2}=n}(-1)^{x_{1}} .
$$

As $H(-m)$ consists of the single class [1, 1, $\left.m^{*}\right]$, by Dirichlet's theorem [4], [5] we have

$$
R_{\left[1,1, m^{*}\right]}(n,-m)=2 \sum_{d \mid n}\left(\frac{-p}{d}\right)=2 \sum_{d \mid N}\left(\frac{-p}{d}\right) .
$$

Thus

$$
R_{\left[4,2, m^{*}\right]}(n,-4 m)=\sum_{d \mid N}\left(\frac{-p}{d}\right)+\frac{1}{2} \sum_{x_{1}^{2}+x_{1} x_{2}+m^{*} x_{2}^{2}=n}(-1)^{x_{1}} .
$$

As $[1,0, m],\left[4,2, m^{*}\right]$ and $\left[4,-2, m^{*}\right]$ comprise the three classes of $H(-4 m)$, and $n$ is coprime with the conductor of the discriminant $-4 m$, namely 2 if $m \neq 27$ and 6 if $m=27$, by Dirichlet's formula we have

$$
\begin{aligned}
R_{[1,0, m]}(n,-4 m)+R_{\left[4,2, m^{*}\right]}(n,-4 m) & +R_{\left[4,-2, m^{*}\right]}(n,-4 m) \\
& =2 \sum_{d \mid n}\left(\frac{-4 m}{d}\right)=2 \sum_{d \mid N}\left(\frac{-p}{d}\right) .
\end{aligned}
$$

Clearly

$$
R_{\left[4,2, m^{*}\right]}(n,-4 m)=R_{\left[4,-2, m^{*}\right]}(n,-4 m)
$$

so that

$$
\begin{aligned}
R_{[1,0, m]}(n,-4 m) & =2 \sum_{d \mid N}\left(\frac{-p}{d}\right)-2 R_{\left[4,2, m^{*}\right]}(n,-4 m) \\
& =2 \sum_{d \mid N}\left(\frac{-p}{d}\right)-2\left(\sum_{d \mid N}\left(\frac{-p}{d}\right)+\frac{1}{2} \sum_{x_{1}^{2}+x_{1} x_{2}+m^{*} x_{2}^{2}=n}(-1)^{x_{1}}\right) \\
& =-\sum_{x_{1}^{2}+x_{1} x_{2}+m^{*} x_{2}^{2}=n}(-1)^{x_{1}},
\end{aligned}
$$

completing the proof.

4. Concluding remarks. The following result is part of $[7$, Theorem 2a]. It does not follow from Theorem 1.

Corollary 9. Let $n=2^{\alpha} 17^{\beta} N$, where $(N, 34)=1$. Then

$$
R_{[1,0,17]}(n,-68)=0 \quad \text { if } N \equiv 3(\bmod 4) .
$$

This result is the special case $a=1, b=0, c=17, d=-68$ of the following elementary result. 
Theorem 2. Let $n=2^{\alpha} N$, where $(N, 2)=1$. Let $K=[a, b, c] \in H(d)$. If

$a \equiv c \equiv 1(\bmod 2), \quad a-c \equiv b \equiv 0(\bmod 4), \quad a-b-c \equiv 0(\bmod 8)$,

then

$$
R_{K}(n, d)=0 \quad \text { if } N \equiv a+2(\bmod 4) .
$$

Proof. Suppose that there exist integers $x$ and $y$ such that

$$
n=a x^{2}+b x y+c y^{2} .
$$

If $4 \mid n$ then

$$
x^{2}+y^{2} \equiv a^{2}\left(x^{2}+y^{2}\right) \equiv a\left(a x^{2}+b x y+c y^{2}\right)=a n \equiv 0(\bmod 4)
$$

so that $x \equiv y \equiv 0(\bmod 2)$. Hence, dividing out powers of 4 in $n$, we deduce that there exist $X \in \mathbb{Z}$ and $Y \in \mathbb{Z}$ such that

$$
a X^{2}+b X Y+c Y^{2}= \begin{cases}N & \text { if } 2 \mid \alpha, \\ 2 N & \text { if } 2 \nmid \alpha .\end{cases}
$$

If $2 \mid \alpha$ then

$X^{2}+Y^{2} \equiv a^{2}\left(X^{2}+Y^{2}\right) \equiv a\left(a X^{2}+b X Y+c Y^{2}\right) \equiv a N \equiv a(a+2) \equiv 3(\bmod 4)$, which is impossible.

If $2 \nmid \alpha$ then $X \equiv Y(\bmod 2)$ and

$$
\begin{aligned}
X^{2}+Y^{2} & \equiv a^{2}\left(X^{2}+Y^{2}\right) \equiv a\left(a X^{2}+(b+c) Y^{2}\right) \equiv a(2 N+b Y(Y-X)) \\
& \equiv 2 a N \equiv 2 a(a+2) \equiv 6(\bmod 8),
\end{aligned}
$$

which is impossible.

This completes the proof that $R_{[a, b, c]}(n, d)=0$ for $N \equiv a+2(\bmod 4)$.

Applying Theorem 2, we obtain

Corollary 10. Let $n=2^{\alpha} 41^{\beta} N$, where $(N, 82)=1$. Then

$$
R_{[1,0,41]}(n,-164)=0 \quad \text { if } N \equiv 3(\bmod 4) .
$$

Corollary 11. Let $n=2^{\alpha} 41^{\beta} N$, where $(N, 82)=1$. Then

$$
R_{[5, \pm 4,9]}(n,-164)=0 \quad \text { if } N \equiv 3(\bmod 4) .
$$

Corollary 12. Let $n=2^{\alpha} 7^{\beta} N$, where $(N, 14)=1$. Then

$$
R_{[1,0,49]}(n,-196)=0 \quad \text { if } N \equiv(-1)^{\beta-1}(\bmod 4) \text {. }
$$

\section{References}

[1] F. van der Blij, Binary quadratic forms of discriminant -23, Indag. Math. 14 (1952), 498-503.

[2] D. A. Buell, Binary Quadratic Forms, Springer, New York, 1989. 
[3] N. A. Hall, The number of representations function for binary quadratic forms, Amer. J. Math. 62 (1940), 589-598.

[4] J. G. Huard, P. Kaplan and K. S. Williams, The Chowla-Selberg formula for genera, Acta Arith. 73 (1995), 271-301.

[5] P. Kaplan and K. S. Williams, On a formula of Dirichlet, Far East J. Math. Sci. 5 (1997), 153-157.

[6] G. A. Lomadze, On the representation of numbers by binary quadratic forms, Tbiliss. Gos. Univ. Trudy Ser. Mekh.-Mat. Nauk 84 (1962), 285-290 (in Russian).

[7] - On the representation of numbers by positive binary diagonal quadratic forms, Mat. Sb. (N.S.) 68 (110) (1965), 282-312 (in Russian); English transl.: Amer. Math. Soc. Transl. (2) 82 (1969), 85-122.

[8] - The representation of numbers by certain binary quadratic forms, Izv. Vyssh. Uchebn. Zaved. Mat. 11 (1970), 71-75.

[9] H. Muzaffar and K. S. Williams, A restricted Epstein zeta function and the evaluation of some definite integrals, Acta Arith. 104 (2002), 23-66.

[10] T. V. Vephvadze [T. V. Vepkhvadze], The representation of numbers by positive Gaussian binary quadratic forms, Sakharth. SSR Mecn. Akad. Moambe 56 (1969), $277-280$ (in Russian).

[11] - The representation of numbers by positive binary quadratic forms with odd discriminant, ibid. 58 (1970), 29-32 (in Russian).

[12] - , The representation of numbers by positive Gaussian binary quadratic forms, Sakharth. SSR Mecn. Akad. Math. Inst. Shrom. 40 (1971), 21-58 (in Russian).

[13] - The representation of numbers by certain binary quadratic forms, Thbilis. Univ. Shrom. A 1 (137) (1971), 17-24 (in Russian).

[14] - , The representation of numbers by positive binary quadratic forms of odd discriminant, Sakharth. SSR Mecn. Akad. Math. Inst. Shrom. 45 (1974), 5-40 (in Russian).

[15] - , The number of representations of numbers by genera of positive binary quadratic forms, ibid. 57 (1977), 29-39 (in Russian).

[16] - General theta-functions with characteristics and exact formulae for binary quadratic forms, Bull. Georgian Acad. Sci. 154 (1996), 341-347.

[17] V. G. Zhuravlev, Review of [16], Math. Rev. 99m:11046.

Département de Mathématiques

Université de Nancy I

54506 Vandœuvre-lès-Nancy, France

E-mail: pierre.kaplan@wanadoo.fr
School of Mathematics and Statistics

Carleton University

Ottawa, Ontario, Canada K1S 5B6

E-mail: williams@math.carleton.ca 